(c) 2010 IEEE. Personal use of this material is permitted. Permission from IEEE must be obtained for all other uses, in any current or future media, including reprinting/republishing this material for advertising or promotional purposes, creating new collective works, for resale or redistribution to servers or lists, or reuse of any copyrighted component of this work in other works. 


\section{Haemodynamic Effect of Coronary Angulations on Subsequent Development of Coronary Artery Disease: A Preliminary Study}

\author{
Thanapong Chaichana and Zhonghua Sun \\ Department of Imaging and Applied Physics, \\ Curtin University, \\ Perth, Western Australia, 6845, Australia \\ 14132256@student.curtin.edu.au,z.sun@curtin.edu.au
}

\author{
James Jewkes \\ Fluid Dynamics Research Group, School of Civil and \\ Mechanical Engineering, Curtin University, \\ Perth, Western Australia, 6845, Australia. \\ J.Jewkes@curtin.edu.au
}

\begin{abstract}
The aim of this study is to investigate the hemodynamic effect of the angulations in the left coronary bifurcation on subsequent development of coronary artery disease. Eight 3D left coronary artery models were generated based on the anatomical details and simulated for numerical analysis. The angulations at the left coronary bifurcation were simulated with angles ranging from $120^{\circ}, \mathbf{1 0 5}^{\circ}, \mathbf{9 0}^{\circ}, 7^{\circ}, \mathbf{6 0}^{\circ}, \mathbf{4 5}^{\circ}$, $30^{\circ}$ to $15^{\circ}$. Computational fluid dynamic models were produced for analysis of flow velocity, wall pressure and wall shear stress. Our results showed that wide angled models produced low wall shear stress and high wall pressure at the left coronary bifurcation regions, whereas, flow pattern was more smooth and laminar with narrow angled models than those with wide angle models. Our analysis indicates the flow-field correlation between coronary angulation and development of atherosclerosis. Future studies are required to analyse the realistic coronary models from patients' data with different degree of coronary stenosis.
\end{abstract}

Keywords-Coronary artery disease, Angulation, Blood flow, Flow velocity, Plaque, Simulation.

\section{INTRODUCTION}

Coronary artery disease (CAD) is the major cause of death in western countries. The most common cause of CAD is atherosclerosis which is caused by the presence of plaques on the artery wall, resulting in the lumen stenosis. Plaques have been particularly involved in blood clot and blocks blood stream to myocardium. This occurs when the coronary plaques suddenly rupture; if a clot cannot be treated in time, then the heart muscle will be impaired due to ischemic changes, leading to ischemia or myocardial infarction or necrosis [1]. Therefore, an early detection and diagnosis of CAD is particularly important for reduction of the mortality and subsequent complications [1].

The natural history of coronary plaque is dependent not only on the formation and progression of atherosclerosis, but also on the vascular remodelling response. If the local wall shear stress is low, a proliferative plaque will form. Local inflammatory response will stimulate the formation of socalled "vulnerable plaque" which is prone to rupture with superimposed thrombus formation. The vast majority of these inflamed high-risk vulnerable plaques cannot be detected by anatomic imaging and myocardial perfusion imaging. Since the progression and development of vulnerable plaque is associated with low wall shear stress and the presence of expansive remodelling, measurement of these characteristics in vivo will enable risk stratification for the entire coronary circulation [2].

The wall shear stress (WSS), wall pressure and blood flow changes in human body cannot be measured directly on blood vessels, CFD can provide alternative ways to diagnose CAD [3]. The WSS factor in coronary artery is known as the significant role playing in the early formation of CAD [4]. In addition, the prediction of coronary disease with the normal coronary artery and WSS as the local vessels wall demonstrates anatomical section predisposed for atherosclerosis development [5].

CFD allows for efficient and accurate computations of hemodynamic features of both normal and abnormal situations in the cardiovascular system, in vivo simulation of coronary artery flow changes [3]. CFD is different from medical imaging visualisation as medical imaging techniques such as coronary angiography or computed tomography angiography provide anatomic alterations of the coronary artery wall due to presence of plaques, thus assessing the degree of lumen stenosis. In contrast, CFD analysis enables hemodynamic changes of the coronary artery, even before the plaques are actually formed in the artery wall or occlude the vessels. Therefore, to some extent, CFD allows an early detection of coronary artery disease and improves understanding the progression of plaques which are considered paramount importance to clinical treatment. This study aims to describe the characteristics of hemodynamic phenomena in simulated coronary models with variable angulations. The assumption of this is different angles in the left coronary bifurcation could affect the blood wall and will provide variable effects on flow analysis to the coronary artery, thus the proposed research will serve the purpose of identifying patients with potential risk of developing coronary artery disease.

iVEC 'The hub of advanced computing in Western Australia' 


\section{MATERIALS AND METHODS}

\section{A. Anatomical details of Left Coronary Artery}

The computed tomographic (CT) data of left coronary artery anatomy was used in this study to generate the mathematical models. The left coronary artery (LCA) branches consist of the left main stem (LMS), left anterior descending (LAD) and left circumflex (LCX), as shown in Figure 1. The LCA has an angulation between the LAD and LCX, showed that perpendicular angle (Figure 1). Thus changing the angles to various degrees will affect the subsequent hemodynamic flow patterns. Simulation of different angles between these two branches was performed in this study. Figure 2 shows the variable angles that were simulated in left coronary artery models.

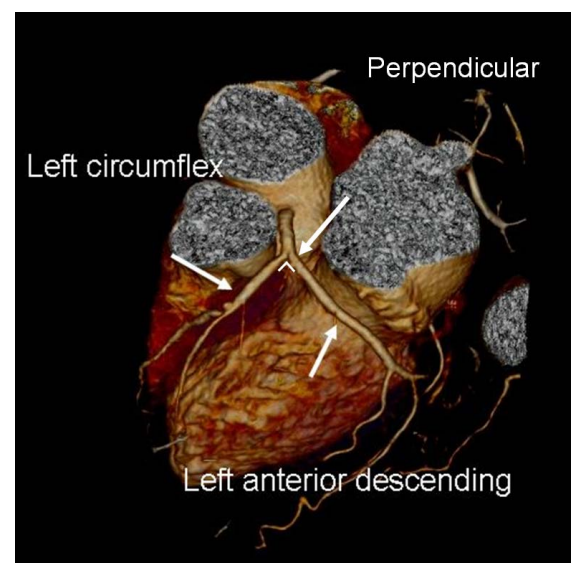

Figure 1. LCA branches on 3D CT visualisation image

\section{B. Measurement of CT volumetric data}

The DICOM (digital imaging and communication in medicine) files from CT scan were transferred to a computer workstation provided with Analyze version 7.0 (AnalyzeDirect, Inc., Lexana, KS, USA) for creation of three-dimensional left coronary artery (LCA) models. The volume model was segmented by using the semi-automatic method with a CT number thresholding technique $[6,7]$ and manual editing was used in some slices to delete the artefacts and soft tissues. The three-dimensional volumetric dimensions of LCA model have been measured, as shown in Table 1.

TABLE I. THE MEASUREMENTS OF NATURE LCA MODEL

\begin{tabular}{|c|c|c|c|}
\hline \multicolumn{2}{|c|}{ Diameter of LCA (mm) } & \multicolumn{2}{c|}{$\begin{array}{c}\text { Distance between bifurcation } \\
\text { to distal/proximal (mm) }\end{array}$} \\
\hline LMS & 3.0 & Proximal LMS & 35 \\
\hline LAD & 2.0 & Distal LAD & 25 \\
\hline LCX & 1.5 & Distal LCX & 20 \\
\hline
\end{tabular}

LMS-left main stem

\section{Generation of left coronary Models}

The surface model was generated based on the anatomic details of LCA in Section II-A and II-B, referencing the LCA anatomy in Figure 1 and the parameters in Table 1 by using
CATIA V5 R18 (Dassault Systemes, Inc., Suresnes, France). The surface model was then converted into the solid model, as shown in Figure 3. According to The $90^{\circ}$ model, other seven models were generated by varying the degree of the $90^{\circ}$ model between LCX and LAD, as shown in Figure 2 and all of models were saved as STP type to generate mesh models.

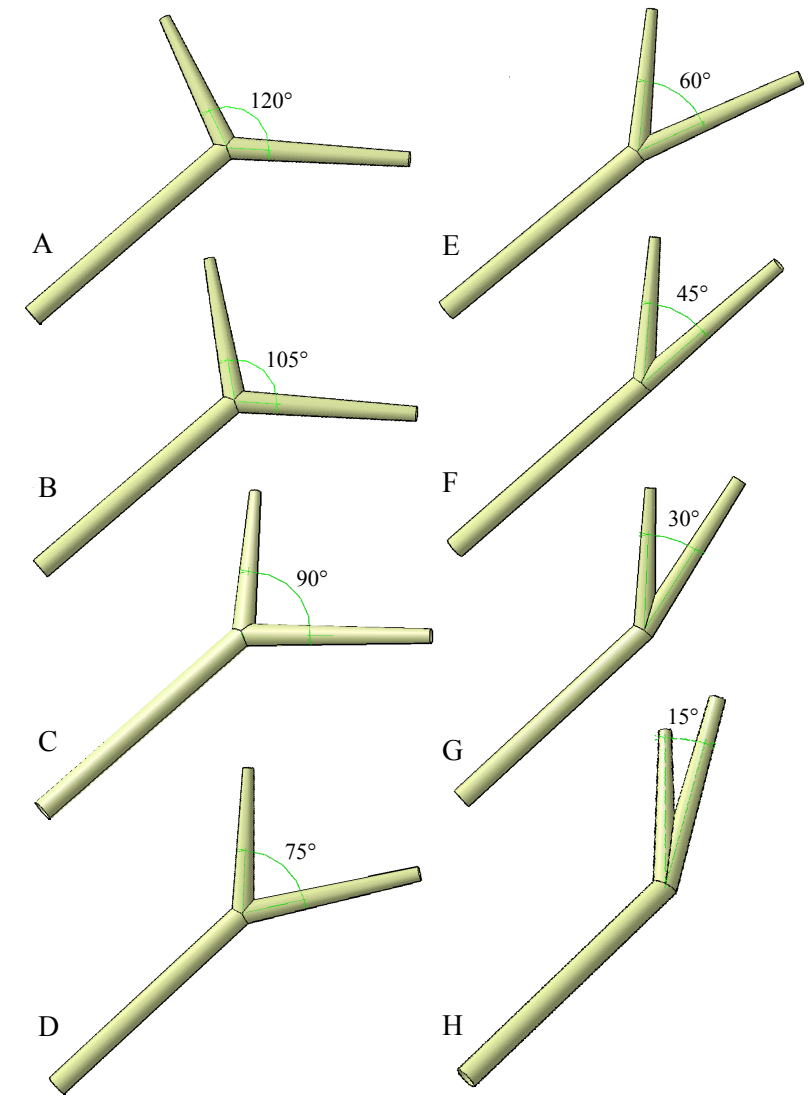

Figure 2. Diagrams showing variable angles of $\mathrm{LCA}, 120^{\circ}(\mathrm{A}), 105^{\circ}(\mathrm{B})$, $90^{\circ}(\mathrm{C}), 75^{\circ}(\mathrm{D}), 60^{\circ}(\mathrm{E}), 45^{\circ}(\mathrm{F}), 30^{\circ}(\mathrm{G})$ and $15^{\circ}(\mathrm{H})$

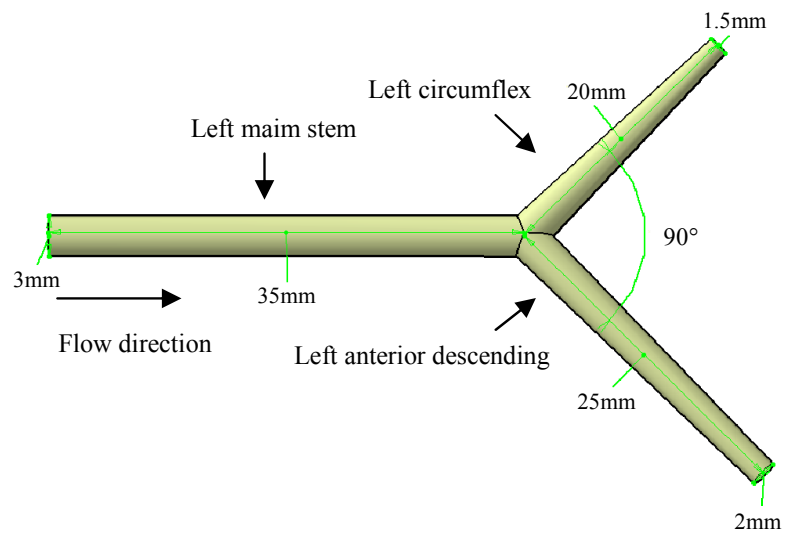

Figure 3. The $90^{\circ}$ model based on the anatomic details 
D. CFD simulation in left coronary artery

\section{1) Generation of Mesh Models}

The eight three-dimensional computer aided design (CADD) models of left coronary were used for generation of mesh models for performance of CFD simulation. In addition, the CADD models were produced using the tetrameshing and three prism layers method, which is suitable for the actual computation. Details of meshing elements are shown in Table 2. The mesh models were generated with the ANSYS ICEM CFD version 12 (ANSYS, Inc., Canonsburg, PA, USA), as referred to in previous experimental studies [8, 9, 10]. Finally, the eight mesh models were saved as GTM type to be use for CFD computation.

TABLE II. THE DETAILS OF MESHING ELEMENTS

\begin{tabular}{|c|c|c|c|}
\hline \multicolumn{4}{|c|}{ The 6 LCA models } \\
\hline Models & Angles & Tetra-mesh & 3 Prism layers \\
\hline $\mathrm{A}$ & $120^{\circ}$ & 365517 & 103695 \\
\hline $\mathrm{B}$ & $105^{\circ}$ & 369218 & 99504 \\
\hline $\mathrm{C}$ & $90^{\circ}$ & 354827 & 97677 \\
\hline $\mathrm{D}$ & $75^{\circ}$ & 357502 & 99153 \\
\hline $\mathrm{E}$ & $60^{\circ}$ & 366925 & 103941 \\
\hline $\mathrm{F}$ & $45^{\circ}$ & 364552 & 104106 \\
\hline $\mathrm{G}$ & $30^{\circ}$ & 358038 & 98988 \\
\hline $\mathrm{H}$ & $15^{\circ}$ & 330427 & 92517 \\
\hline
\end{tabular}

\section{2) Application of physiological parameters}

In order to ensure that our analysis reflected the realistic simulation of in vivo conditions, application of the physiological parameters was considered for the threedimensional numerical analysis. The transient simulation was performed, and accurate hemodynamic rheological and material properties were used in this study, as referred to in previous studies [8]. According to Bertolotti et al [9], flow rate graph at LCA was reconstructed by developing algorithm using Matlab (MathWorks, Inc. Natick, MA, USA) for creation of Fourier series graph as shown in Figure 4 . The boundary conditions were determined by pulsatile velocity at left main stem (inlet) and opening pressure with left anterior descending (outlet) and left circumflex (outlet). Accurate physiological parameters were applied with a blood density of $1060 \mathrm{~kg} / \mathrm{m} 3$, blood viscosity of $0.0027 \mathrm{~Pa} \mathrm{~s}$. The blood flow and wall were assumed to be Laminar flow and no-slip conditions. Blood was assumed to be a Newtonian $[10,4]$ and incompressible fluid [11].

\section{3) Performance of CFD computation}

To ensure that our results are valid, the simulation was performed by using ANSYS CFX version 12 (ANSYS, Inc.) which was equipped with the Microsoft Windows XP 32-bit, $4 \mathrm{MB}$ RAM and running on the dual core $2.4 \mathrm{GHz}$ CPU. A left coronary artery in normal flow situation is governed by the Navier-Stokes equations. The CFD simulation was run for 32 time steps, each converged to 100 iterations, representing 1.6 seconds of pulsatile flow. The computed time consumption for each LCA model was approximately one and half hour. This computation was performed similar to an in vivo condition by referring to previous experiments $[12,13,14]$. The flow pattern, flow velocity, wall pressure, and wall shear stress were calculated and visualised using ANSY CFD-Post version 12 (ANSYS, Inc.).

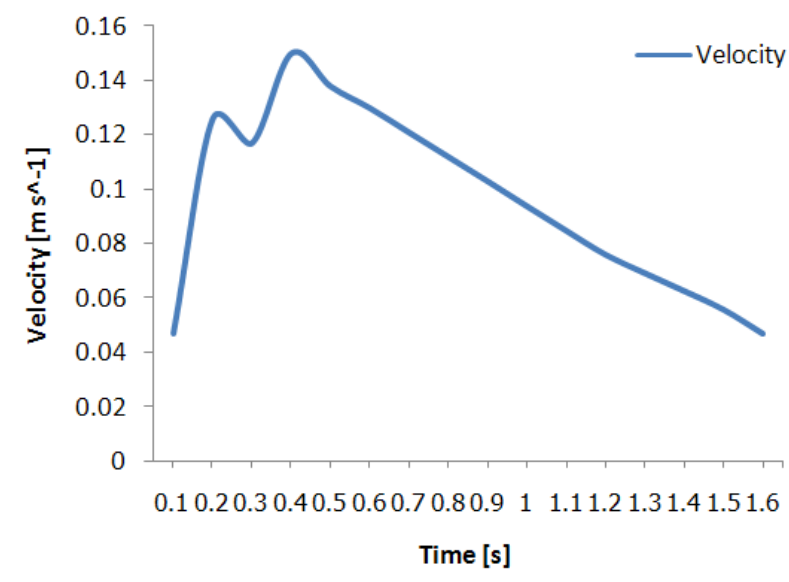

Figure 4. Pulsatile velocity at left coronary artery

\section{RESULTS}

Eight left coronary models were performed with CFD analysis under the in vivo conditions during the cardiac cycles (systolic and diastolic phases). The analysis results showed the stream connection between hemodynamic effects and the simulations of angled models. Figure 4 shows the highest velocity peak reached during systolic phase at time $0.4 \mathrm{sec}$ Figure 5 shows velocity measured with different coronary models with small and wide angled models demonstrating laminar flow pattern, while wide angled models presenting turbulent flow, respectively.

Low WSS appears at the bifurcation regions where the left coronary main stem branches into left anterior descending and left circumflex. Figure 6 shows that the WSS was found to reduce apparently in wide angled models when compared with the narrowed models and this phenomenon is particularly obvious in the systolic phase.

Similarly, the wall pressure has been affected to some extent with the change of angulations in different models. Figure 7 demonstrates that the wall pressure decreased from wide angled models to narrowly angled models. This is especially apparent in the model with a $15^{\circ}$ angulation as the results showed that the wall pressure reduced significantly when the blood flows through from the left main stem to the LAD and LCx braches. 


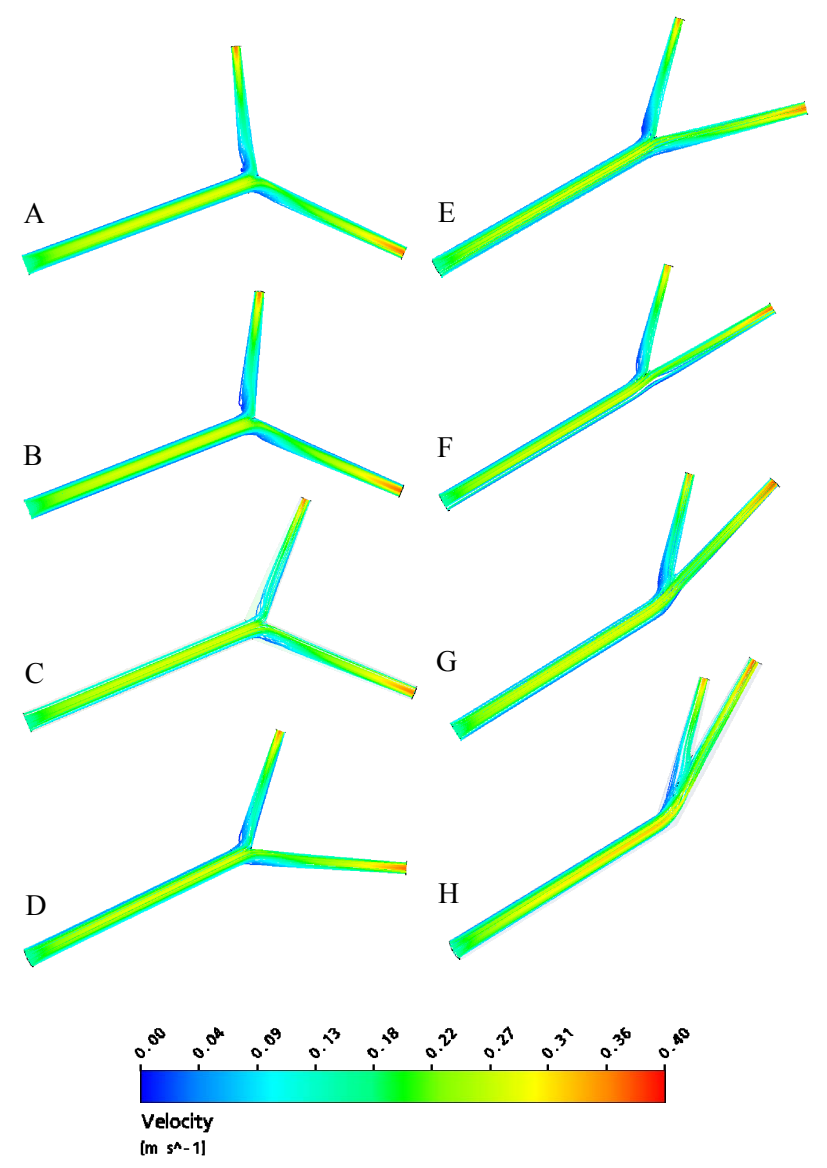

Figure 5. Velocity showing variable angles of LCA, A-H: measured at systolic peak $0.4 \mathrm{sec}$

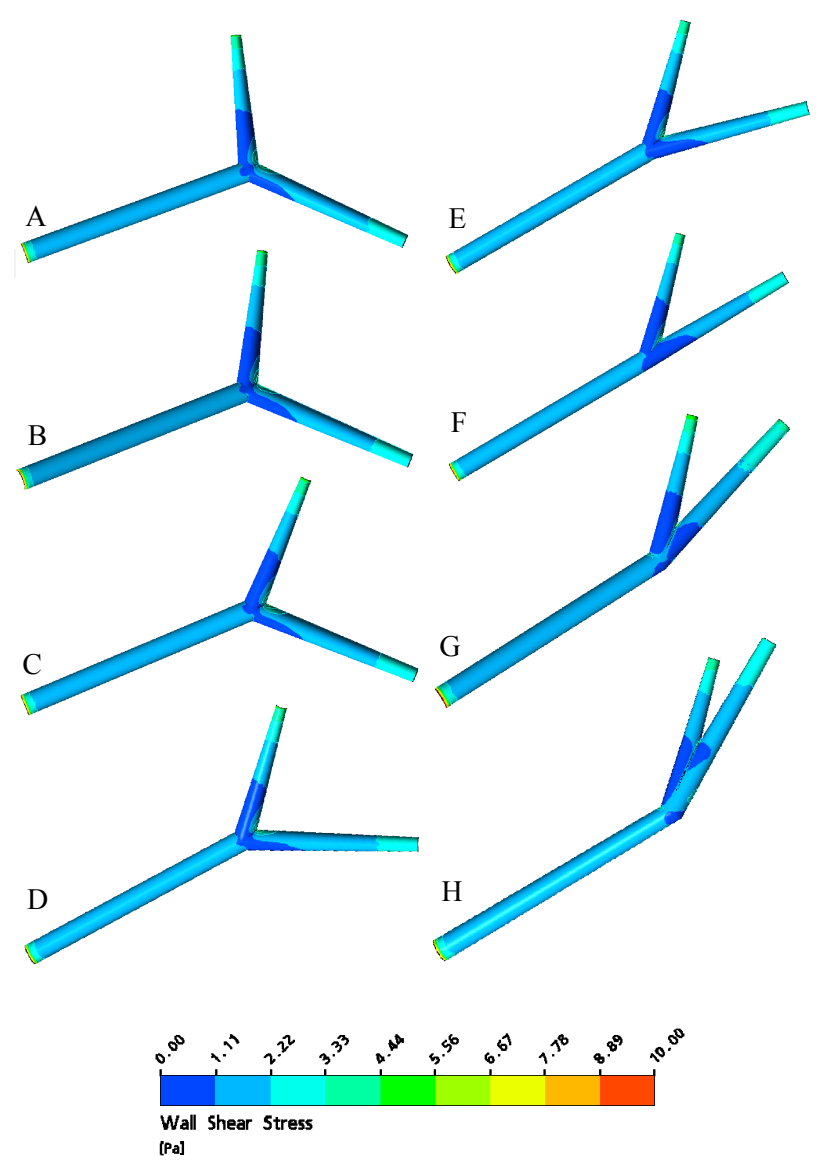

Figure 6. Wall shear stress with variable angles of LCA, A-H: measured at systolic peak $0.4 \mathrm{sec}$

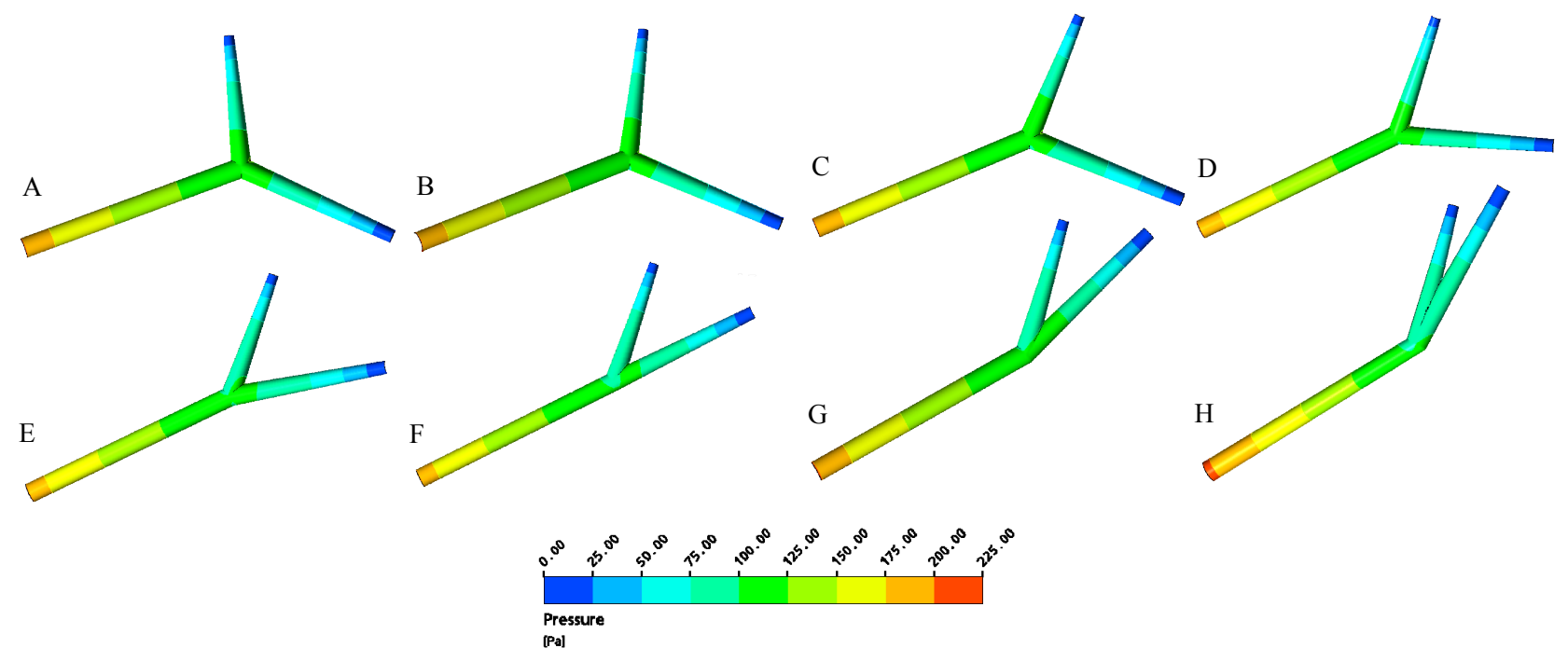

Figure 7. Wall pressure with variable angles at $120^{\circ}(\mathrm{A}), 105^{\circ}(\mathrm{B}), 90^{\circ}(\mathrm{C}), 75^{\circ}(\mathrm{D}), 60^{\circ}(\mathrm{E}), 45^{\circ}(\mathrm{F}), 30^{\circ}(\mathrm{G})$ and $15^{\circ}(\mathrm{H})$ 


\section{DISCUSSION}

This preliminary study is valuable for improving understandding the development of atherosclerosis of coronary artery disease. The study results based on the different angula-tions of coronary models show the stream connection between angulations and change of wall shear stress and flow pattern.

Coronary disease is mainly caused by atherosclerosis and it is a chronic, inflammatory and fibroproliferative disease mostly involving large- and medium-sized arteries [15, 16]. It has been reported that atherosclerotic lesions form at specific regions of the arterial tree, such as in the vicinity of branch points, the outer wall of bifurcation, and the inner wall of curvatures, where disturbed flow occurs [17]. Local factors such as hemodynamic forces play a major role in the regional localization of atherosclerosis [18]. These local hemodynamic forces include flow-generated shear stress and wall pressure with wall shear stress playing the most fundamental role in atherosclerosis, This is confirmed in our study as low wall shear stress occurred at wide angled models, indicating the tendency to form atherosclerosis.

Current imaging modalities such as coronary CT or invasive coronary angiography cannot provide adequate information about the microenvironment of the coronary arteries, especially the local hemodynamic changes, despite superior capability of providing anatomical details by these techniques. In contrast, CFD analysis offers opportunities to study the effect of variable situations such as different angles at left bifurcation and subsequent flow changes. Therefore, we believe our preliminary study provides insight into the development of atherosclerosis of coronary artery disease.

One of the limitations of the study is that the simulated models are rigid rather than elastic. Simulation of coronary models according to fluid-structure interaction would allow us to draw robust conclusion. Another limitation of this study is lack of realistic models. Thus, future studies are required to study the local hemodynamic environment responsible for individual plaque behaviour and natural history of coronary artery disease based on patient-specific models.

In conclusion, our results show that there is strong relationship between wide angulation in the left coronary bifurcation and development of atherosclerosis. Further studies based on realistic models are needed to verify our initial results.

\section{ACKNOWLEDGEMENT}

The PhD scholarship awarded by E-Medicine centre, Western Australia is greatly appreciated.

\section{REFERENCES}

[1] Australian Institute of Health and Welfare, "The tenth biennial health report of the Australian Institute of Health and Welfare," Cat. no. AUS 73. Canberra: AIHW, 2006

[2] F. Ribicki, S. Melchionna, D. Mitsouras, A. Coskun, A. Whitmore, M. Steigner, L. Nallamshetty, F. Welt, M. Bernaschi, M. Borkin, J. Sircar, E. Kaxiras, S. Succi, P. Stone and C. Feldman, "Prediction of coronary artery plaque progression and potential rupture from 320-detector row prospectively ECG-gated single heart beat CT angiography: Lattice Boltzmann evaluation of endothelial shear stress," International Journal of Cardiovascular Imaging, vol. 25, pp. 289-299, 2009.

[3] S. Shanmugavelayudam, D. Rubenstein and W. Yin, "Effect of geometrical assumptions on numerical modelling of coronary blood flow under normal and disease conditions," Journal of Biomechanical Engineering, vol. 132, pp. 1-8, 2010.

[4] B. Johnston, P. Johnston, S. Corney and D. Kilpatrick, "Non-Newtonian blood flow in human right coronary arteries: steady state simulations," Journal of Biomechanics, vol. 37, pp. 709, 2004.

[5] V. Soulis, T. Farmakis, G. Giannoglou and G. Louridas, "Wall shear stress in normal left coronary artery tree," Journal of Biomechanics, vol. 39, pp.742-743, 2006.

[6] Z. Sun, J. Winder, B. Kelly, P. Ellis, D. Hirst, "CT virtual intravascular endoscopy of abdominal aortic aneurysms treated with suprarenal endovascular stent grafting," Abdom Imaging, vol. 28, pp. 580-587, 2008.

[7] Z. Sun, J. Winder, B. Kelly, P. Ellis, P. Kennedy, D. Hirst, "Dianostic value of CT virtual intravascular endoscopy in aortic stent grafting", Journal of Endovascular Therapy, vol. 11, pp. 13-25, 2004.

[8] T. Frauenfelder T, Lotfey $\mathrm{M}$, Boehm $\mathrm{T}$ and Wildermuth $\mathrm{S}$, "Computational fluid dynamics: hemodynamic changes in abdominal aortic aneurysm after stent-graft implantation," CardioVascular and Intervenlional, vol. 29, no. 4, pp. 724, 2006.

[9] C. Bertolotti, Deplanoa V, Fuseri J and Dupouyb P, "Numerical and experimental models of post-operative realistic flows in stenosed coronary bypasses," Journal of Biomechanics, vol. 34, pp. 1049-1064, 2001.

[10] B. Johnston, Johnston P, Corney S and Kilpatrick D, "Non-Newtonian blood flow in human right coronary arteries: Transient simulations," Journal of Biomechanics, vol. 39, pp. 1116-1128, 2006.

[11] A. Borgh, Wood N, Mohiaddin R, and Xu X, "Fluid-solid interaction simulation of flow and stress pattern in thoracoabdominal aneurysms: A patient-specific study", Journal of Fluids and Structures, vol. 24, no. 2, pp. 270-28, 2008.

[12] Z. Sun and T. Chaichana, "Fenestrated Stent Graft Repair of Abdominal Aortic Aneurysm: Hemodynamic Analysis of the Effect of Fenestrated Stents on the Renal Arteries," Korean Journal Radiology, vol. 11, no. 1, pp. 95-106, 2010.

[13] Z. Sun and T. Chaichana, "Investigation of the Hemodynamic Effect of Stent Wires on Renal Arteries in Patients with Abdominal Aortic Aneurysms Treated with Suprarenal Stent-Grafts," CardioVascular and Interventional Radiology, vol. 32, pp. 647-657, 2009.

[14] Z. Sun, T. Chaichana, B. Mwipatayi and C. Ng, "Hemodynamic effect of calcified plaque on blood flow in carotid artery disease: A preliminary study", IEEE proceedings of the 3rd International conference on Bioinformatics and Biomedical Engineering, Beijing, pp. 1-4, 2009.

[15] R. Ross, "Atherosclerosis-an inflammatory disease," The New England Journal of Medicine, vol. 340, pp. 115-126, 1999.

[16] GK. Hansson, "Inflammation, atherosclerosis, and coronary artery disease," The New England Journal of Medicine, vol. 352, pp. 16851695, 2005.

[17] PA. VanderLaan, CA. Reardon and GS. Getz, "Site specificity of atherosclerosis: site-selective responses to atherosclerotic modulators," Arteriosclerosis, Thrombosis, and Vascular Biology, vol. 24, pp. 12-22, 2004.

[18] AM. Malek, SL. Alper and S. Izumo, "Hemodynamic shear stress and its role in atherosclerosis," Journal of the American Medical Association, vol. 282, pp. 2035-2042, 1999. 